Cinémas

Revue d'études cinématographiques

Journal of Film Studies

\title{
Métatextualité filmique : vers une métacritique
}

\section{Martin Lefebvre}

Volume 5, numéro 3, printemps 1995

Cinélekta 1

URI : https://id.erudit.org/iderudit/1001153ar

DOI : https://doi.org/10.7202/1001153ar

Aller au sommaire du numéro

Éditeur(s)

Cinémas

ISSN

1181-6945 (imprimé)

1705-6500 (numérique)

Découvrir la revue

Citer cet article

Lefebvre, M. (1995). Métatextualité filmique : vers une métacritique. Cinémas, 5(3), 165-178. https://doi.org/10.7202/1001153ar

\section{Résumé de l'article}

À partir de la notion de métatextualité telle que développée par Genette dans Palimpseste, cet article s'interroge sur la façon dont le commentaire représente le texte qu'il commente. La sémiotique peircéenne sera mobilisée pour examiner le mode d'être du texte commenté à l'intérieur du commentaire. Ce dernier assure ainsi la circulation d'un modèle du texte commenté, modèle susceptible de s'imposer et d'acquérir une certaine valeur culturelle à titre d'interprétation, de représentation, ou même de reproduction d'un corpus. 


\title{
Métatextualité filmique: vers une métacritique ${ }^{1}$
}

\section{Martin Lefebvre}

\begin{abstract}
RÉSUMÉ
A partir de la notion de métatextualité telle que développée par Genette dans Palimpseste, cet article s'interroge sur la façon dont le commentaire représente le texte qu'il commente. La sémiotique peircéenne sera mobilisée pour examiner le mode d'être du texte commenté à l'intérieur du commentaire. Ce dernier assure ainsi la circulation d'un modèle du texte commenté, modèle susceptible de s'imposer et d'acquérir une certaine valeur culturelle à titre d'interprétation, de représentation, ou même de reproduction d'un corpus.
\end{abstract}

\section{ABSTRACT}

Following Genette's notion of metatextuality as developped in Palimpseste, this article questions the way texts are represented in critical commentary. Peircean semiotic is called upon to examine the mode of being of the commented text within the commentary. Thus, it is shown that commentary creates and circulates a model of the commented text. This model can eventually impose itself and acquire a certain cultural capital as interpretation, representation, or reproduction of a corpus.

On a déjà soulevé le caractère dialogique du discours critique, qui inscrit dans son tissu textuel un autre texte dont il se fait le commentaire. Comme le disait Barthes: "[...] la critique est discours sur un discours [...] c'est un métalangage" (p. 255). Dans sa taxinomie des relations textuelles, Gérard Genette 
nomme "métatextualité" cette relation " [...] qui unit un texte à un autre texte dont il parle", et qui constitue, "[...] par excellence, la relation critique" (p. 10). Mais ce qui a retenu l'attention des théoriciens de la littérature jusqu'ici, c'est surtout l'attitude intertextuelle d'une critique moderne et non la qualité intertextuelle sui generis du métadiscours.

Cette perspective informe le travail de Leyla Perrone-Moisés, à qui revient, à ma connaissance, d'avoir étudié la première l'intertextualité critique. Perrone-Moisés refuse de considérer la critique "traditionnelle" comme une forme véritable d'intertextualité puisqu'elle ne constitue pas une écriture et conserve la frontière entre le discours et le métadiscours. Seuls quelques critiques comme Barthes, Butor et Blanchot franchissent cette frontière par leur pratique d'écriture, ce qui permet " [...] l'apparition d'un discours véritablement intertextuel " : " [...] dans une critique-écriture il y aura un véritable dialogue parce que la nouvelle parole sera en condition d'égalité par rapport à celle qui lui sert de pré-texte; le critique ne se placera plus devant l'autre texte comme un suiveur, mais comme un poursuiveur d'ambiguïtés, c'est-à-dire comme un écrivain" (p. 377). En définitive, cette critique-écriture rend tout simplement inopérante la distinction entre textes poétiques et textes critiques.

On retrouve plus ou moins la même perspective dans l'étude de Michel Charles sur la pratique de la lecture critique. L'auteur montre comment celle-ci se distingue des autres genres du métadiscours, tels l'essai critique ou le traité théorique, par l'intégration et la mise au premier plan "d'un réseau de citations originellement contiguës " et susceptibles d'être manifestées in extenso, et par son apparition à des moments historiques déterminés, marqués par une critique de la glose et un "retour au texte" (p. 131). Il s'agit d'une double définition qui suppose un rapport étroit entre des choix méthodologiques et stylistiques: l'usage de la citation in extenso renvoie à une intention critique précise: la volonté d'utiliser le commentaire pour servir le texte et non l'inverse, ce qui distingue la lecture critique des autres genres métatextuels, qui ne sont pas étrangers, bien entendu, à la citation. Cet usage de la citation favorise d'une part l'apparition d'intratextes lorsque le commentaire cite ou fait allusion au 
texte cité in extenso et d'autre part, la constitution d'un foyer intertextuel lorsque le commentaire importe des citations extérieures au texte commenté, histoire de montrer que ce dernier "[...] est déjà un montage citationnel» (p. 149). Source d'intratextes et d'intertextes, "la lecture critique", conclut l'auteur, "[...] n'est sans doute pas une forme nécessaire de l'activité métatextuelle, mais qu'elle se manifeste comme telle est un signe de la modernité" (p. 151).

Mais malgré les mérites des études menées par L. PerroneMoisés et M. Charles pour une éventuelle typologie du discours critique, il faut reconnaître que le caractère dialogique du commentaire lui-même s'y trouve évacué. L'intertextualité perd ici sa valeur de principe opératoire du discours pour devenir une propriété générique du discours critique. Les deux auteurs, par conséquent, limitent, chacun à leur manière, le dialogisme à un rapport homodiscursif. M. Charles adopte la perspective qu'il retrouve chez Montaigne, en refusant d'attribuer le statut de texte au discours critique: ce dernier sert simplement de foyer aux relations intratextuelles et intertextuelles que propose le montage des diverses citations. Perrone-Moisés, de son côté, utilise la notion d'écriture pour gommer la différence entre le texte poétique et le texte critique, afin que s'estompe toute hiérarchie entre les textes qui composent le système intertextuel. Or, si ce nivellement de l'intertextualité nous renseigne quant aux dispositions d'une certaine critique moderne, force est de reconnaître qu'on fait violence inutilement au principe de la circulation des textes, principe qui octroie au discours critique sa dimension intertextuelle sui generis.

Je propose donc qu'on revienne au point de départ, tel que souligné par Perrone-Moisés au début de son étude lorsqu'elle reconnaît qu' " [...] en principe, la critique a toujours été intertextuelle [...]. Il s'est toujours agi d'écrire un texte sur un autre texte, un texte qui dialogue avec un autre. Ainsi, même dans le cas le plus simple (évidemment hypothétique, comme toutes les "formes simples"), il y a dans le discours critique l'entrecroisement de deux textes, le texte analysé et le texte analysant " (p. 372). Or, pour spécifier cette relation, il importe d'interroger le mode d'être du texte commenté à l'intérieur du commentaire. 
Une première observation, c'est que le métatexte critique manifeste ouvertement sa relation avec le texte qu'il commente. À cet égard, l'usage de la citation, comme le montre Michel Charles, implique la présence effective du texte commenté dans le commentaire. Mais il serait faux de croire qu'il ne s'agit là que d'une simple reproduction du texte cible ou, comme le mentionne Perrone-Moisés, que d'une "simple addition de textes" (p. 373). Bien au contraire, l'espace métatextuel transforme nécessairement le texte commenté par un processus complexe de représentation. Inutile de revenir ici sur la leçon borgesienne pour montrer comment la citation même la plus directe implique toujours une transformation: la nouvelle citée in extenso par Roland Barthes à la fin de $S / Z$ ne correspond pas à Sarrasine tel que ce texte apparaît dans La Pléiade, qui, lui, n'est pas une citation.

En fait, le texte commenté est toujours sémiotisé dans et par le commentaire pour devenir l'objet d'une (ou de plusieurs) représentation(s). Pour élucider les avatars de cette représentation métatextuelle, je ferai appel à la théorie sémiotique développée par Charles S. Peirce. On sait qu'Antoine Compagnon a déjà utilisé le modèle peircien pour examiner, dans une perspective interdiscursive (ou intertextuelle), la valeur sémiotique de la citation, qu'il définit dans son ouvrage La Seconde Main comme un énoncé répété et une énonciation répétante. Deux raisons motivent, chez Compagnon, le choix de la sémiotique peircienne: d'abord, la nature plus flexible du signe triadique, plus maniable que la structure binaire du signe saussurien pour la description des systèmes de signes en général; ensuite, une certaine parenté avec la conception du signe de l'ancienne rhétorique (notion de sèmeion telle qu'elle s'oppose à celle de symbolon chez Aristote), "[...] cas unique d'un signe qui se définit d'emblée au niveau du discours et non de la langue» (p. 60).

La citation, explique Compagnon, consiste en une relation sémiotique où un système textuel (le representamen) en représente un autre (son objet) par voie d'une valeur de répétition (l'interprétant). Chaque système textuel est composé d'au moins un énoncé et d'une énonciation, ce qui permet à la citation de revêtir plusieurs visages selon les composantes qu'elle met en jeu 
dans ses divers usages (diverses interprétances). Se limitant au rapport representamen/objet, l'auteur montre que la citation sera évaluée tantôt comme symbole (la citation d'un théorème mathématique, par exemple), tantôt comme indice (mode habituel de la citation dans le discours critique) ou comme icône (le pastiche, la parodie, la citation implicite, etc. $)^{2}$.

La citation, toutefois, ne représente qu'une seule des stratégies de répétition du discours critique. Le métatexte peut également représenter le texte commenté de façon moins directe en utilisant le résumé, l'allusion, la paraphrase, etc. Dans certains domaines comme le cinéma, la performance théâtrale ou musicale, le discours critique ne peut d'ailleurs pas citer le texte commenté. Contrairement au champ littéraire, où les pratiques métadiscursives du commentaire reposent sur le même matériau que le texte commenté, ces domaines composent avec un discours critique dont le matériau est hétérogène au texte commenté : celui-là est un texte, celui-ci est un film, une performance, un édifice, etc. Impossible donc, comme le remarquait Raymond Bellour, de citer le film à l'intérieur du discours critique: "[...] le texte filmique est un texte introuvable, parce que c'est un texte incitable" (p. 37). Dans la mesure où la citation, au sens strict du terme, implique la répétition d'un énoncé et d'une énonciation, l'activité critique en matière de cinéma suppose une transformation profonde de la relation entre le commentaire et le texte commenté, ce qui donne à l'analyse filmique une complexion particulière:

L'analyse du film [...] ne cesse ainsi de mimer, d'évoquer, de décrire; elle ne peut, avec une sorte de désespoir de principe, que tenter une concurrence effrénée avec l'objet qu'elle cherche à comprendre. Elle finit, à force de chercher à le saisir et à le ressaisir, par être le lieu même d'un dessaisissement perpétuel. [...] C'est pourquoi elles [les analyses filmiques] semblent toujours un peu fictives: jouant sur un objet absent, et sans jamais pouvoir, puisqu'il s'agit de le rendre présent, se donner les moyens de la fiction, tout en devant les emprunter. L'analyse du film ne cesse de remplir un film qui ne cesse de fuir; elle est par excellence le tonneau des Danaïdes (Bellour, p. 40). 
La différence entre la critique littéraire et la critique cinématographique est de taille: le film n'est pas présent dans le commentaire sur le mode de la citation, mais de la paraphrase, de l'allusion, ou mieux, de la traduction (de l'image en mouvement au mot et à la photo); ce qui suppose une transformation encore plus radicale, quoique souvent inavouée, du texte cible ${ }^{3}$. L'analyse du film, par un jeu de présence/absence involontaire, doit donc traduire le texte filmique afin d'assurer son élucidation et sa reconstruction à l'intérieur du texte critique ${ }^{4}$, ce qui nécessite déjà un travail de représentation ou d'interprétation ${ }^{5}$.

Cette traduction est assurée tant bien que mal par un ensemble d'outils analytiques développés par la théorie du cinéma afin de décrire certains aspects du film, et particulièrement de la bande-image ${ }^{6}$. L'analyse du film se référera aux notions de plan et de séquence à partir desquelles on élabore la description du montage, du récit (actions, agents, temporalité, spatialité), des images (éclairage, couleur, mouvements d'appareils), etc. ${ }^{7}$. Ces notions permettent de transcrire et d'inscrire, avec plus ou moins de succès, le filmique dans le langage et dans l'image fixe. D'une certaine manière, le succès du discours critique dépend de cette sémiotisation: le commentaire critique porte sur un objet du monde - le film - dont il est, en quelque sorte, une représentation.

À cet égard, c'est d'abord sous le mode de la ressemblance que le commentaire doit, par principe, enchâsser et représenter le texte commenté: si le texte-commenté-enchâssé ne ressemble pas au texte-commenté-objet-du-monde, la valeur référentielle du commentaire peut être remise en cause. Le lecteur dira tout simplement: "C'est un mauvais commentaire puisque je n'y reconnais pas l'objet commenté." C'est ce qu'on peut appeler la dimension iconique de l'intertextualité critique ${ }^{8}$; dimension qui est liée pragmatiquement au succès de la fonction explicative de l'analyse du film et de laquelle découle la dimension indicielle mentionnée plus haut dans la typologie de Compagnon. Ainsi, la traduction du texte filmique dans le commentaire, dans la mesure où elle sert d'exemplum, pourra devenir un indice dans le processus d'explication du film, ce qui implique une relation de contingence entre le commentaire et le texte commenté. Tout 
dépend, comme on le voit, du point du vue qu'on adopte face au signe? .

Mais une fois l'identité du texte-commenté-enchâssé reconnue, il y a, parallèlement, un autre sens selon lequel le commentaire représente le texte qu'il commente par le biais de l'interprétation. Ainsi, lorsque Raymond Bellour affirme que Psycho remet en jeu deux structurations psychiques, la névrose et la psychose, et que "[...] la femme, sujet de la névrose, devient l'objet de la psychose dont l'homme est le sujet " (p. 298), le commentaire se place en relation d'interprétance face au texte commenté et à son interprétation: le discours critique représente le film à titre de représentation d'un certain contenu. Soumis à l'analyse sémiotique, ce rôle bien conventionnel du discours critique peut être illustré par le schéma suivant:

discours critique

(interprétant)

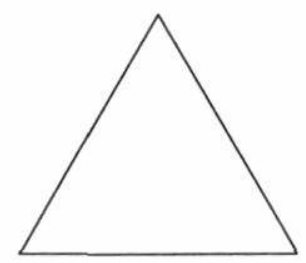

texte commenté

interprétation

(representamen)

(objet)

Ce diagramme représente la fonction métatextuelle du commentaire: le discours critique enchâsse un texte qu'il commente et interprète. Cette opération nécessite les trois termes de la sémiose, le representamen, l'objet et l'interprétant, qui s'étayent selon une logique de la représentation réciproque: le representamen représente son objet par voie de l'interprétant (le film représente un contenu tel qu'assuré par le commentaire); l'interprétant représente le representamen dans sa relation de représentation avec l'objet (le commentaire représente le film dans sa relation au contenu); l'interprétant est déterminé par le representamen (le commentaire est en un sens "produit " par le film); le representamen et l'interprétant sont des représentations 
différentes de l'objet (le film et le commentaire représentent tous les deux le contenu, et se représentent ainsi mutuellement). C'est ce que Peirce appelle la triadicité authentique.

Or, chaque commentaire critique à propos d'un film peut générer des interprétations plus ou moins différentes selon les diverses méthodes d'interprétation du discours critique et selon le principe fondamental de l'inépuisement sémantique des textes et de la sémiose illimitée ${ }^{10}$. À partir d'un même film, différents discours critiques participent à la génération de diverses interprétations. On peut représenter la multiplicité des interprétations de la manière suivante:

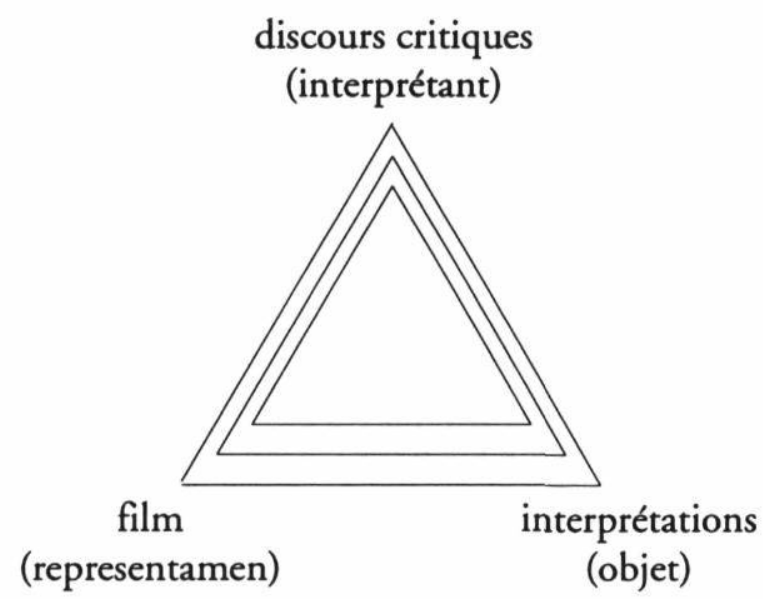

Les trois triangles illustrent trois parcours interprétatifs différents. Leur non-congruence au niveau du representamen évite la réification de ce dernier, qu'il ne faut pas identifier à l'objet matériel qu'est le film: le representamen, c'est le film tel qu'interprété par l'interprétant et non le film en soi; chaque sémiose risque donc d'avoir un representamen différent.

En ce qui concerne le discours critique, la tendance rhétorique est de représenter le representamen comme un signe de loi, en sorte qu'on peut être tenté de le mettre au profit de ce que Peirce appelle l'argument. Comme le souligne Umberto Eco: "[...] in scientific discovery one figures out laws through the mediating discovery of many further facts; and in text interpretation one identifies new relevant facts by presupposing certain 
general (intertextual) laws» (p. 205). Or, dans les faits, la nature du savoir produit - qui ne fait pas l'unanimité chez les différents herméneutes d'un même texte " — nous amène à considérer qu'il s'agit plutôt d'un dicisigne: le commentaire représente le film comme une proposition attributive, que d'autres interprétations pourront vérifier ou falsifier par la suite ${ }^{12}$.

Mais si le discours critique sert d'interprétant au texte filmique, il sert également de representamen à un "modèle " du film. Gérard Genette a bien compris ce rôle du commentaire lorsqu'il examine la "lecture" qu'a fait Freud d' Edipe (le texte mythique ou la tragédie de Sophocle et non le personnage):

Non seulement, donc, il [Freud] propose une interprétation, par exemple du texte de Sophocle, mais encore il l'interprète comme une récriture censurée, et ce faisant il en suggère à son tour une récriture décensurée où l'enchaînement des conduites, immotivé dans l'hypotexte [la tragédie] puisque déterminé de l'extérieur par des oracles qui sont des ordres, se trouve maintenant déterminé de l'intérieur par un motif inconscient (p. 375).

Comme l'explique Genette, le commentaire de Freud propose ici un nouveau texte, une version différente de la tragédie grecque, qui met à jour les motivations réprimées d' $E$ dipe. Non seulement le commentaire freudien interprète-t-il le texte de Sophocle, mais il suggère également la représentation d'un nouvel CEdipe. De la même manière, lorsque Raymond Bellour affirme, toujours à propos de Psycho, que le vol de Marion Crane répond à la fois à " [...] l'agression sociosexuelle dont elle est métaphoriquement l'objet de la part du "millionnaire" dans le bureau de Cassidy" et "[...] à l'agression dont elle se sent l'objet de la part de Sam, dans la clandestinité honteuse de la chambre d'hôtel" (p. 300), l'auteur réécrit le film en octroyant à l'héroïne des motivations qui restent indéterminées dans le film; on assiste alors à un montage intertextuel qui met en jeu le film d'Hitchcock et sa réécriture: d'un côté, le commentaire sert d'interprétant au film, de l'autre côté il représente une nouvelle version, un modèle du film.

Or, si cette dérivation intertextuelle relève d'une évidence dans le cas des lectures psychanalytiques ou "symptomales" 
(telles que popularisées par Althusser), elle ne s'y limite pas; les lectures barthésienne de Balzac ou greimassienne de Maupassant montrent bien comment le commentaire suggère toujours une espèce de modèle du texte commenté: chaque herméneutique crée sa propre version, son propre texte, qui se superpose à l'interprétation (le contenu "découvert») du texte commenté, aussi "fidèle" soit-elle. Même le commentaire le plus descriptif devra choisir l'objet de sa description (en fonction du récit? de l'image? du son ?) et donc interpréter minimalement le texte qu'il commente: la constitution du modèle n'y est que moins flagrante, un point c'est tout. En sorte que la représentation métatextuelle d'un texte suggère toujours la représentation d'un nouveau texte. Par conséquent, le métatexte se trouve à l'origine d'une nouvelle triade :

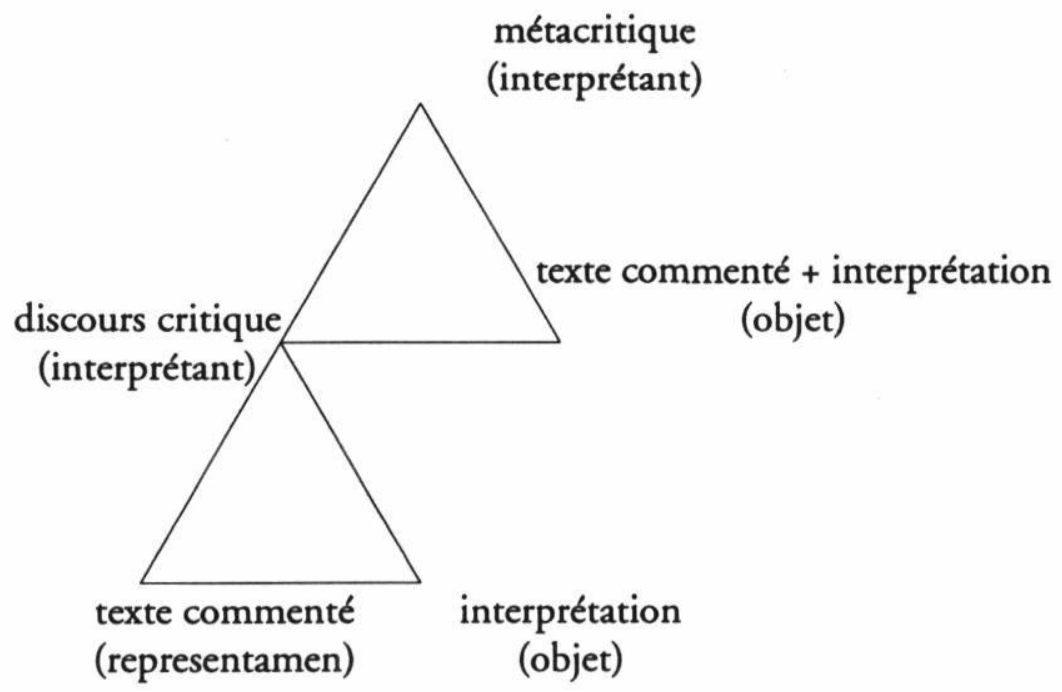

La nouvelle triade présente le processus de la semiosis illimitée, tel que postulé par Peirce dans cette célèbre définition du signe :

Un signe ou representamen est un premier qui entretient avec un second appelé son objet une relation triadique si authentique qu'elle peut déterminer un troisième appelé son interprétant, à entretenir avec son objet la même relation triadique qu'il entretient luimême avec ce même objet. Cette relation triadique est 
authentique, c'est-à-dire que ses trois membres sont liés ensemble par elle de telle façon qu'elle ne se ramène pas à un quelconque complexe de relations dyadiques. [...] Le troisième $[\ldots]$ doit avoir une deuxième relation triadique dans laquelle le representamen, ou plutôt la relation du representamen à son objet, soit son propre objet (l'objet du troisième), et doit pouvoir déterminer un troisième à cette relation. Tout ceci doit également être vrai des troisièmes du troisième et ainsi de suite indéfiniment (p. 147).

Cette nouvelle triade nous permet d'interpréter les divers modèles qui se dégagent de la circulation et de l'interprétation d'un corpus à l'intérieur des discours critiques. Il s'agit d'une troisième forme de représentation du texte commenté à l'intérieur du commentaire: pour la métacritique (critique de la critique), le commentaire - par son choix de textes commentés et par les méthodes d'interprétation qu'il utilise - (re)présente un certain modèle du corpus qu'il commente. C'est en quoi le cinéma d'Alfred Hitchcock - pour prendre un exemple célèbre - fera apparaître soit un simple technicien virtuose, soit un moraliste, soit un "métaphysicien, platonicien et catholique" ou encore un "psychologue des profondeurs" comme le dit Deleuze, qui préfere néanmoins un Hitchcock inventeur de "l'image-relation" (p. 270-277). De même, la lecture féministe de l'œuvre hitchcockienne ${ }^{13}$ représente un corpus qui differe de celui qui se dégage de la lecture générique $^{14}$, etc. Autant de modèles, de textes, construits grâce à des interprétations dont le but est de "faire du sens" à partir des films eux-mêmes, quelle que soit la stratégie interprétative employée ${ }^{15}$. C'est en quoi il serait possible d'identifier divers modèles d'une œuvre cinématographique au fil des avatars de sa circulation à l'intérieur de différents métatextes, qui correspondent, en termes sémiotiques, à autant de sinsignes indiciaires dicents.

Pour la métacritique, il ne s'agit donc pas de savoir si tel ou tel métatexte représente un modèle fiable d'une œuvre ou même de savoir s'il s'agit d'une bonne ou d'une mauvaise interprétation, ou encore de découvrir quels critiques ont raison et lesquels ont tort. Il s'agit plutôt de reconnaître comment la circulation métatextuelle participe au développement de différentes versions d'un corpus donné. Ces versions sont susceptibles de 
changer selon les différentes conceptions, les différents choix théoriques qui orientent le discours critique et circulent dans l'espace culturel au même titre que les autres modèles intertextuels qui peuvent accompagner le même corpus ${ }^{16}$. Certains modèles se cristallisent par la fréquence à laquelle ils circulent et finissent par acquérir une sorte de valeur culturelle au sein d'une communauté interprétative à titre d'interprétation, de représentation ou même de reproduction d'un corpus. De cette manière, on peut dire que ces modèles concourent pour une place au sein d'une sorte de musée imaginaire du cinéma dans un sens proche de son acception chez Malraux ${ }^{17}$ et assistent au développement de la culture filmique ${ }^{18}$ : c'est-à-dire qu'ils constituent une sélection, un choix, un répertoire participant à l'essor d'un savoir sur le cinéma. La métacritique, en ce sens, a pour tâche d'étudier la formation, la circulation et l'accréditation de ces modèles dans l'espace culturel.

\section{University of Alberta}

\section{NOTES}

1 Cet article s'inscrit dans un travail plus vaste sur le rôle joué par l'intertextualité et la métatextualité eu égard à la place occupée par l'œuvre d'Alfred Hitchcock dans la culture filmique.

2 Malgré sa valeur heuristique, il faut reconnaître que cette typologie reste bien précaire: le signe peircien n'est pas réifiable et sa nature change selon le point de vue qu'on adopte.

3 Il semblerait, à ce titre, qu'il faille compter sur l'exclusion de la lecture critique au sein des discours critiques des études filmiques. Cette conclusion mérite cependant d'être nuancée. Si la matière filmique résiste à la citation, force est de constater néanmoins que la place des études sur le cinéma dans le domaine des humanités et l'influence considérable des études littéraires sur leur développement académique a rangé l'analyse du film (ou analyse textuelle) du côté de la lecture critique: non seulement les approches structuralistes ont-elles formellement donné au film son statut " textuel ", mais on y retrouve également l'ensemble des stratégies théoriques de la lecture critique telle qu'elle s'élabore dans le champ littéraire, comme en témoignent les exemples donnés par M. Charles, $S / Z$ de Barthes ou l'analyse du poème Les Chats par Lévi-Strauss et Jakobson, qui ont généré des tentatives similaires sur des corpus filmiques, en dépit du statut incitable du texte filmique.

4 Contrairement à la lecture critique, toutefois, l'analyse textuelle favorise habituellement le fragment. En posant le principe de l'homogénéité du film classique, le fragment acquiert une valeur métonymique. Voir Raymond Bellour dans L'Analyse du film (Paris: Albatros, 1979) et Jacques Aumont et Michel Marie dans L'Analyse des films (Paris: Nathan, 1988). 
5 Dans le contexte de la sémiotique peircienne, représentation et interprétation peuvent être considérées comme des synonymes.

6 Il s'agit d'une préférence historique de plus en plus contreversée. Voir, entre autres, les ouvrages de Michel Chion, Sarah Kozloff et Elisabeth Weis, de même que les anthologies dirigées par Elisabeth Weis, John Belton et Rick Altman.

7 Voir à ce sujet le travail de synthèse de Jacques Aumont et Michel Marie dans L'Analyse des films.

8 Rappelons ici que pour Peirce, l'icône désigne un des neuf sous-types de signes, qu'il est premier dans la dimension de l'objet (qui est second) et qu'il définit un rapport de ressemblance entre le signe et son objet.

9 Reste qu'il faille néanmoins respecter la logique des catégories qui sous-tend le projet peircien : un premier est toujours une monade, un second est composé d'un premier et d'un second, enfin, un troisième est composé d'un premier, d'un second et d'un troisième. Dans sa dimension iconique, l'intertextualité critique est la représentation d'une qualité, ce qui correspond à la première classe de signe proposée par Peirce: qualisigne iconique rhematique; dans sa dimension indicielle, il s'agit de la quatrième classe de signe : sinsigne indiciaire dicent; le commentaire sert à souligner l'existence de ce qu'il représente, existence sur laquelle repose, en dernière analyse, la valeur référentielle du commentaire.

10 Ce qui ne veut pas dire, bien entendu, que toutes les interprétations soient possibles ou qu'elles soient aléatoires. Voir à ce sujet Umberto Eco, The Limits of Interpretation (Bloomington: Indiana University Press, 1990) et Umberto Eco et Stephan Collini (direction), Interpretation and Overinterpretation (Cambridge: Cambridge University Press, 1992).

11 La notion de "communauté interprétative" développée par Stanley Fish décrit bien les mécanismes qui régissent l'unanimité et la dissension des herméneutes d'un texte. Pour plus de détails, voir Stanley Fish, Is There a Text in This Class: The Authority of Interpretative Communities (Cambridge: Harvard University Press, 1980).

12 La nature complète du signe correspond une fois de plus à un sinsigne indiciaire dicent : le commentaire interprète le film comme le signe de l'existence d'un contenu, c'est pourquoi la relation du representamen à l'objet est indicielle; tel que considéré ici, le film est un signe en vertu de sa singularité, ce qui explique sa valeur de sinsigne.

13 Voir Tania Modleski, The Women Who Knew Too Much (New York: Routledge, 1988).

14 Voir Lesley Brill, The Hitchcock Romance (Princeton : Princeton University Press, 1988).

15 David Bordwell, pour sa part, distingue quatre types de significations dans la lecture d'un film: le sens référentiel de la lecture narrative ou diégétique, le sens explicite de la lecture intentionnelle, le sens implicite de la lecture thématique et le sens réprimé de la lecture symptomale. Voir David Bordwell, Making Meaning (Cambridge: Harvard University Press, 1989), p. 8-9.

16 L'œuvre de Hitchcock, par exemple, a donné lieu a d'innombrables pastiches ou parodies, surtout depuis quelques années. Voir notre article «De la reprise à la figure: intertextualité et culture filmique ", $R S / S I$, vol. 11, n* 2-3 (1991), p. 103-130.

17 On se souviendra que le musée imaginaire de Malraux, qui articule différents savoirs sur l'art, regroupe les artefacts qu'il "exhibe" grâce à des processus de représentation comme la reproduction photographique qui participe à la circulation d'œuvres d'art tout en opérant sur eux certaines transformations. Le discours critique, à sa manière, n'en fait pas moins.

18 Sur la notion de culture filmique, voir « De la reprise à la figure : intertextualité et culture filmique ", $R S / S I$, vol. 11, n* 2-3 (1991). 


\section{OUVRAGES CITÉS}

Barthes, Roland. Essais critiques. Paris: Seuil, 1964.

Bellour, Raymond. L'Analyse des films. Paris: Albatros, 1979.

Charles, Michel. "La lecture critique». Poétique, n³4 (1978).

Compagnon, Antoine. La Seconde main. Paris: Seuil, 1979.

Deleuze, Gilles. Cinéma I. L'Image-mouvement. Paris: Minuit, 1983.

Eco, Umberto et Sebeok, T. (direction). The Sign of Three. Bloomington: Indiana University Press, 1983.

Genette, Gérard. Palimpseste. Paris: Seuil, 1982.

Peirce, Charles S. Ecrits sur le signe. Paris: Seuil, 1978.

Perrone-Moisés, Leyla. "L'intertextualité critique ». Poétique, n 27 (1976). 